\title{
MICROTOMOGRAFIA COMPUTADORIZADA: PRINCÍPIOS DE FUNCIONAMENTO E UTILIZAÇÃO EM AMOSTRAS BIOLÓGICAS
}

\author{
Paulo José Bastos Queiroz ${ }^{1}$, Anne Karoline Mendes da Silva ${ }^{2}$, Sabrina Lucas Ribeiro \\ de Freitas ${ }^{3}$, Bruno Moraes Assis ${ }^{4}$, Luiz Antônio Franco da Silva ${ }^{5}$ \\ ${ }^{1}$ Médico Veterinário, Mestre em Ciência Animal, Professor no Instituto Federal do \\ Norte de Minas Gerais - Campus Salinas, Salinas-MG, Brasil. \\ Email: paulojose.vet@hotmail.com \\ ${ }^{2}$ Graduanda em Medicina Veterinária no Instituto Federal do Norte de Minas Gerais - \\ Campus Salinas, Salinas-MG, Brasil \\ ${ }^{3}$ Médica Veterinária, Mestre em Ciência Animal, Professora no Instituto Federal \\ Goiano - Campus Urutaí, Urutaí-GO, Brasil. \\ ${ }^{4}$ Médico Veterinário, Mestre em Ciência Animal, Professor no Centro Universitário \\ Una - Unidade Jataí, Jataí-GO, Brasil \\ ${ }^{5}$ Médico Veterinário, Doutor em Ciência Animal, Professor na Universidade Federal \\ de Goiás, Goiânia-GO, Brasil.
}

\section{Recebido em: 06/04/2019 - Aprovado em: 10/06/2019 - Publicado em: 30/06/2019 DOI: 10.18677/EnciBio_2019A90}

\begin{abstract}
RESUMO
A microtomografia computadorizada (microCT) é uma técnica de avaliação de materiais, que utiliza o princípio físico da atenuação de raios $X$ para formação de imagens bidimensionais e tridimensionais. Essa técnica possibilita a visualização tridimensional e em alta resolução de pequenas amostras, por meio da união digital de centenas de cortes transversais. A avaliação pela microCT requer mínimas preparações da amostra e, por ser uma técnica não destrutiva, não ocasiona danos ao material. Diante do crescente uso da microCT, o presente trabalho teve por objetivo fazer uma revisão de literatura sobre os princípios básicos de funcionamento e a utilização da microCT em alguns tipos de amostras biológicas. Durante a revisão de literatura são abordados tópicos sobre aspectos históricos do desenvolvimento da microCT, princípios de funcionamento do equipamento de microCT e aplicação da técnica em alguns tipos de amostras biológicas, tais como ossos, dentes, cartilagens, sistema vascular e fígado de pequenos roedores. Os autores apresentam algumas vantagens da utilização da microCT em pesquisas sobre morfologia de tecidos biológicos, que podem ser realizadas com amostras ex vivo e em experimentos in vivo com pequenos roedores. Espera-se que a divulgação desta técnica amplie as pesquisas com microCT em amostras biológicas no Brasil.
\end{abstract}

PALAVRAS-CHAVE: imagem 3D, microCT, $\mu \mathrm{CT}$. 


\title{
MICRO-COMPUTED TOMOGRAPHY: PRINCIPLES OF OPERATION AND USE IN BIOLOGICAL SAMPLES
}

\begin{abstract}
Micro-computed microtomography (microCT) is a material evaluation technique that uses the principle of X-ray attenuation for the formation of two-dimensional and three-dimensional images. This technique allows the visualization of high-resolution and three-dimensional images of small samples through the digital union of hundreds of cross-sections. In view of the increasing use of microCT, this study aims to review the basic principles of microCT operation and its use in some types of biological samples. During the review are discussed topics on historical aspects of microCT development, principles of microCT equipment operation and application of the technique in some types of biological samples such as bones, teeth, cartilage, vascular system and small rodent liver. The authors present some advantages of using microCT in research on morphology of biological tissue, which can be performed with ex vivo samples and in vivo experiments with small rodents. It is expected that the dissemination of this technique will broaden the research with microCT in biological samples in Brazil.
\end{abstract}

KEYWORDS: 3D image, microCT, $\mu \mathrm{CT}$.

\section{INTRODUÇÃO}

A descoberta dos raios $X$ possibilitou que várias técnicas de avaliação médica surgissem, dentre estas a radiografia (METSÄLÄ; FRIDELL, 2018), a fluoroscopia (ISAIAH; PEREIRA, 2017), a angiografia (ÇIMEN et al., 2016), a mamografia (FREER; WINKLER, 2017) e a tomografia computadorizada (TC) (SCHICHOR et al., 2017). Derivada da TC, a microtomografia computadorizada (microCT) é capaz de produzir imagens em alta resolução de pequenas amostras. Essa técnica possibilita a reconstrução de imagens tridimensionais, que são formadas pela união digital de centenas de secções transversais do material avaliado (BAIRD; TAYLOR, 2017).

A avaliação pela microCT, também denominada de Tomografia Computadorizada de Alta Resolução, requer mínimas preparações da amostra e, por ser uma técnica não destrutiva, não ocasiona danos ao material (DU PLESSIS; BOSHOFF, 2019). O princípio físico para a formação da imagem microtomográfica baseia-se na propriedade dos materiais de atenuação dos raios X (MACHADO et al., 2014), o qual depende da composição e da densidade do material avaliado (DU PLESSIS; BOSHOFF, 2019). A microCT tem sido aplicada em diversas áreas do conhecimento, como: caracterização de materiais (HANKE et al., 2016), arqueologia (ZANOLLI et al., 2017), geologia (JACQUES et al., 2014), engenharia de tecidos (BOERCKEL et al., 2014), odontologia (DALSTRA et al., 2015) e ortopedia (ROBERTO-RODRIGUES et al., 2015).

Diante do crescente uso da microCT para a avaliação dos mais diversos tipos de amostras biológicas, objetivou-se fazer uma revisão de literatura sobre os princípios básicos de funcionamento e a utilização da microCT em amostras biológicas.

\section{ASPECTOS HISTÓRICOS SOBRE O DESENVOLVIMENTO DA MICROCT}

Os raios $X$ foram descobertos acidentalmente pelo físico alemão Wilhelm Conrad Röntgen, no dia 8 de novembro de 1895. Diante dessa descoberta, Röntgen nomeou essas ondas eletromagnéticas de raios $X$, símbolo matemático que indica algo desconhecido (CIERNIAK, 2011). 
Em 1916, o polonês Karol Mayer desenvolveu uma metodologia que é considerada a precursora da tomografia clássica. Esse pesquisador descreveu uma técnica que produzia imagens radiográficas do coração sem a ocorrência de sobreposições, utilizando um emissor de raios $X$ móvel e um chassi radiográfico fixo (LEE; CREAN, 2013). O francês André Edmond Marie Bocage pode ser considerado o pai da tomografia, pois, em 1922, foi o primeiro a descrever o funcionamento básico de um equipamento radiográfico, que movia simultaneamente o emissor de raios $X$ e o filme radiográfico (LEE; CREAN, 2013).

O progresso da computação foi fundamental para o grande desenvolvimento dos exames tomográficos. A união do computador com os raios $\mathrm{X}$ é considerada uma das inovações mais importantes da segunda metade do século $X X$ (HESSENBRUCH, 2002). No início da década de 1970, pesquisadores desenvolveram diversas técnicas de reconstrução digital de imagens tomográficas, que possibilitaram uma nova era no diagnóstico radiológico (LEE; CREAN, 2013). Em 1979, Allan Cormack e Godfrey Hounsfield ganharam o Prêmio Nobel de Fisiologia e Medicina pelo desenvolvimento da TC (BOERCKEL et al., 2014).

A utilização da TC em outras áreas como a engenharia de materiais e a nanotecnologia requeria a formação de imagens de alta resolução e em escala micrométrica. Logo, um grupo de pesquisadores se empenhou no desenvolvimento de equipamentos tomográficos capazes de reconstruírem imagens tridimensionais em dimensões microestruturais (STOCK, 1999). No início da década de 1980, o físico Lee Feldkamp desenvolveu o primeiro microtomógrafo para avaliação de defeitos estruturais de materiais automotivos (BOERCKEL et al., 2014). Em seguida, alguns estudos foram publicados utilizando essa técnica em pequenas amostras biológicas, principalmente em pesquisas com pequenos roedores (ELLIOTT; DOVER, 1984, 1985).

Nos anos que se seguiram, ficou evidente que a microCT revolucionaria as áreas da biologia óssea e biomecânica (BOERCKEL et al., 2014). A partir de então, esse exame de imagem começou a ser utilizado na avaliação de amostras biológicas, principalmente em avaliações ósseas e odontológicas. Dessa forma, houve um grande crescimento no número de publicações que utilizaram a microCT na avaliação dos mais diversos tipos de materiais (SCHAMBACH, et al., 2010). Atualmente, vários sistemas de microCT estão disponíveis no mercado e as inovações tecnológicas continuam aumentando a resolução e aplicabilidade (BOERCKEL et al., 2014).

\section{PRINCÍPIOS DE FUNCIONAMENTO DA MICROCT}

$O$ equipamento de microCT é formado por alguns componentes principais: o emissor microfocal de raios $\mathrm{X}$, o colimador, o suporte para a amostra e o detector de raios $X$. Esse último é constituído por um detector de cintilação e um dispositivo de carga acoplada (CCD) (BOERCKEL et al., 2014) (Figura 1). Inicialmente, em avaliações de amostras rígidas ex vivo, como osso e dente, o fragmento é fixado firmemente a um suporte no interior do microtomógrafo (Figura 2 - A). A avaliação de modelos experimentais in vivo, como pequenos roedores, requer a anestesia geral e contenção do animal em um suporte específico para esse tipo de escaneamento (BEAUCAGE et al., 2016) (Figura 2 - B).

Os raios $X$ são gerados pelo emissor microfocal de radiação, colimados e, na sequência, incidem sobre a amostra, onde parte desses raios é atenuada de acordo com a característica de cada material. $O$ restante da radiação passa pela amostra e atinge o detector de cintilação (CLARK; BADEA, 2014). Ao atingir o detector de 
cintilação, a radiação é transformada em luz visível (fótons), que é identificada pelo CCD. Os fótons de luz ao incidirem no CCD geram um sinal eletrônico, que é digitalizado e enviado ao computador, onde os dados são processados (HOLDSWORTH; THORNTON, 2002; LI et al., 2008). Cada pixel de uma imagem obtida no escaneamento corresponde à média de absorção de fótons pelo detector de cintilação. No processo de reconstrução da imagem tridimensional, o pixel é transformado em voxel, unidade tridimensional capaz de representar a profundidade na imagem microtomográfica (LOPES et al., 2012).

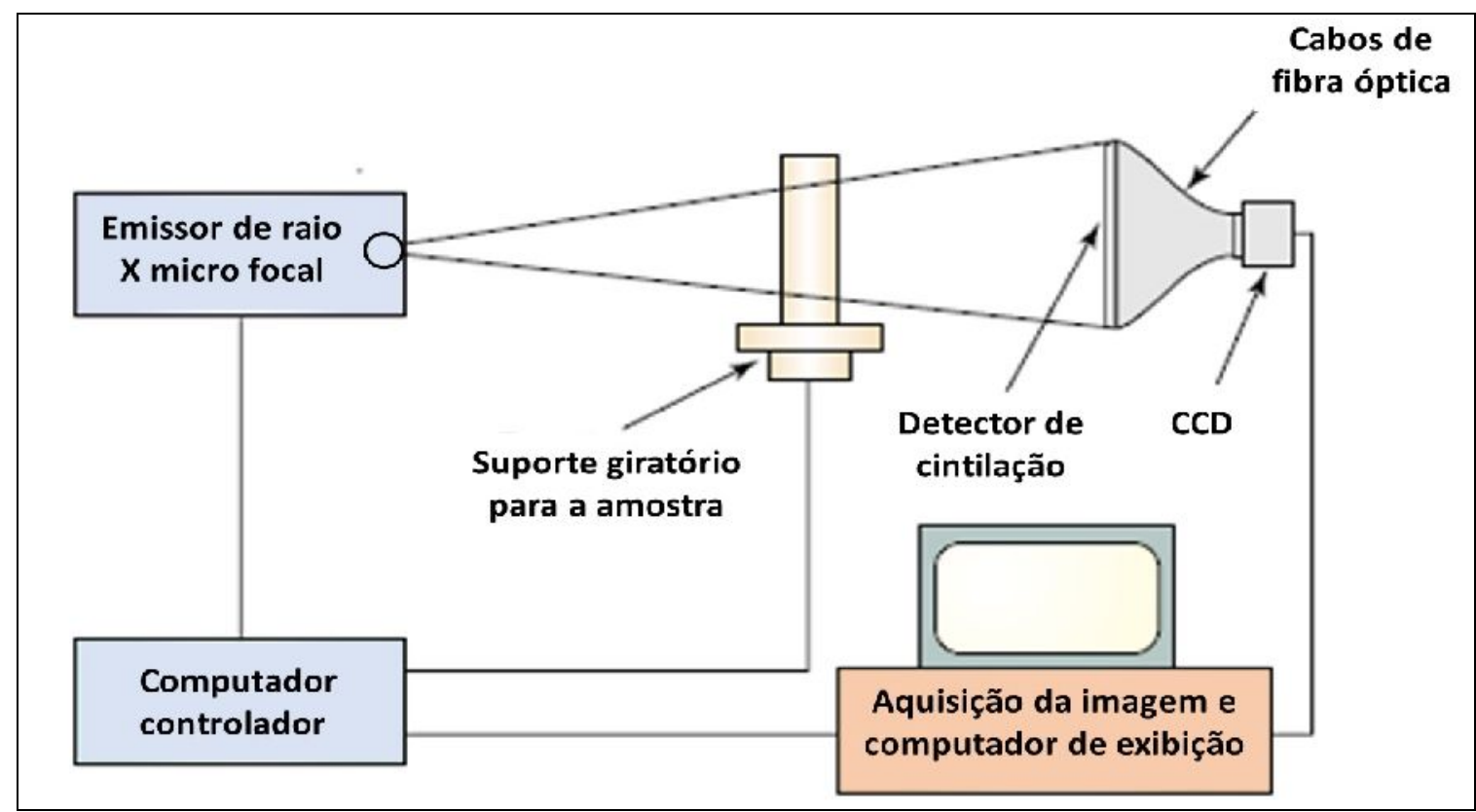

FIGURA 1 - Esquema de um equipamento de microtomografia. A amostra é posicionada no suporte giratório entre o emissor de raio $\mathrm{X}$ e o sensor de cintilação. As projeções radiográficas são obtidas pelo detector de cintilação, que é conectado a um dispositivo de carga acoplada (CCD), que transforma a luz visível em sinal digital. Durante o escaneamento, o computador controlador configura a intensidade de emissão de raios $X$ e a rotação da amostra, possibilitando a obtenção de centenas de projeções em vários ângulos de incidência. Fonte: adaptado de Holdsworth e Thornton (2002).

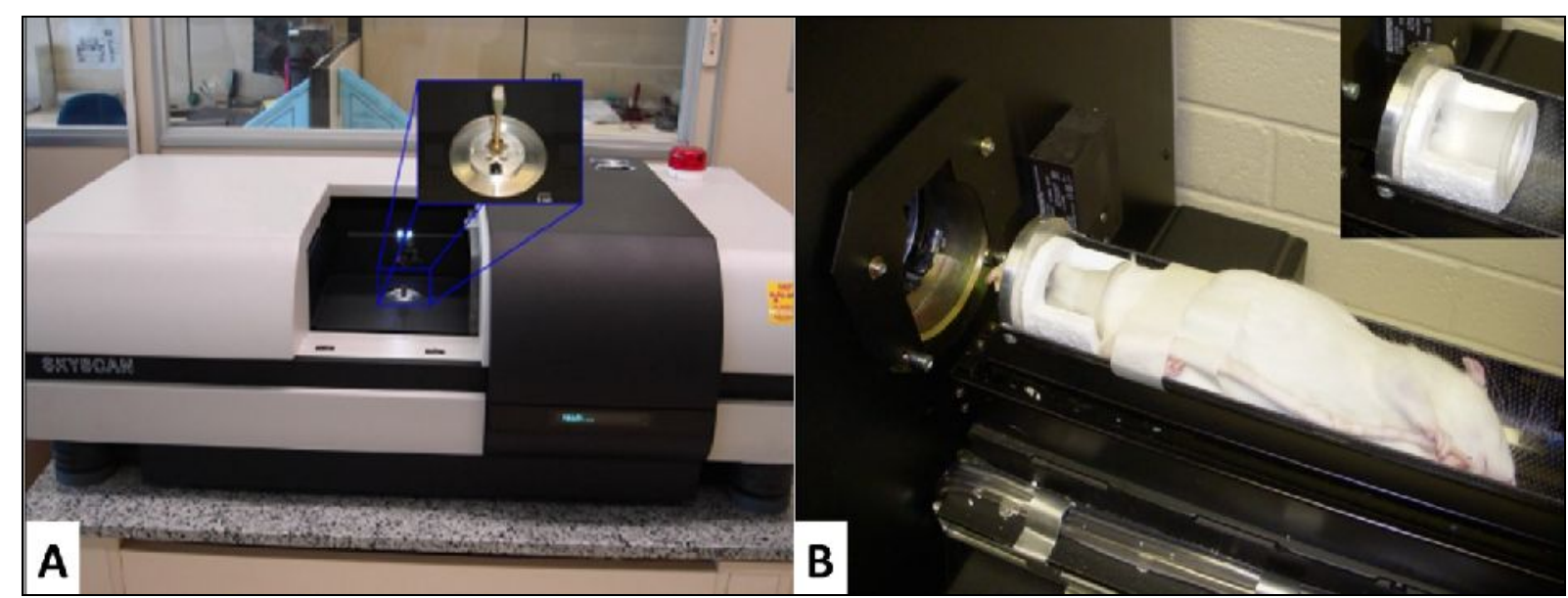

FIGURA 2 - Avaliação microtomografia de amostras biológicas ex vivo e in vivo. (A) Microtomógrafo laboratorial, Skyscan 1172. No detalhe suporte para fixação da amostra. (B) Camundongo anestesiado e fixado no suporte de escaneamento para realização de exame microtomográfico do membro pélvico. Fonte: (A) Fernandes et al. (2016). (B) Mohan et al. (2011). 
O escaneamento microtomográfico produz imagens tridimensionais em resoluções microscópicas pela reconstrução de centenas de imagens bidimensionais, obtidas de múltiplos ângulos em torno da amostra (CLARK; BADEA, 2014). Dessa forma, a microCT gera grande quantidade de gigabytes de dados, os quais são armazenados no hardware do computador. Para a visualização dos cortes bidimensionais e reconstrução dos modelos tridimensionais, as imagens obtidas são carregadas em softwares de análises especializados, preferencialmente em um computador de alto desempenho (BAIRD; TAYLOR, 2017). O software de análise tridimensional une as projeções bidimensionais, baseando-se em um algoritmo matemático de reconstrução pré-estabelecido. Por meio dos softwares de avaliação de imagens, o modelo tridimensional pode ser cortado em qualquer orientação para revelar diferentes pontos de vista da estrutura interna da amostra (BADEA et al., 2008). Na Figura 3 estão representadas as etapas para a obtenção do modelo tridimensional de uma amostra por meio da microCT.

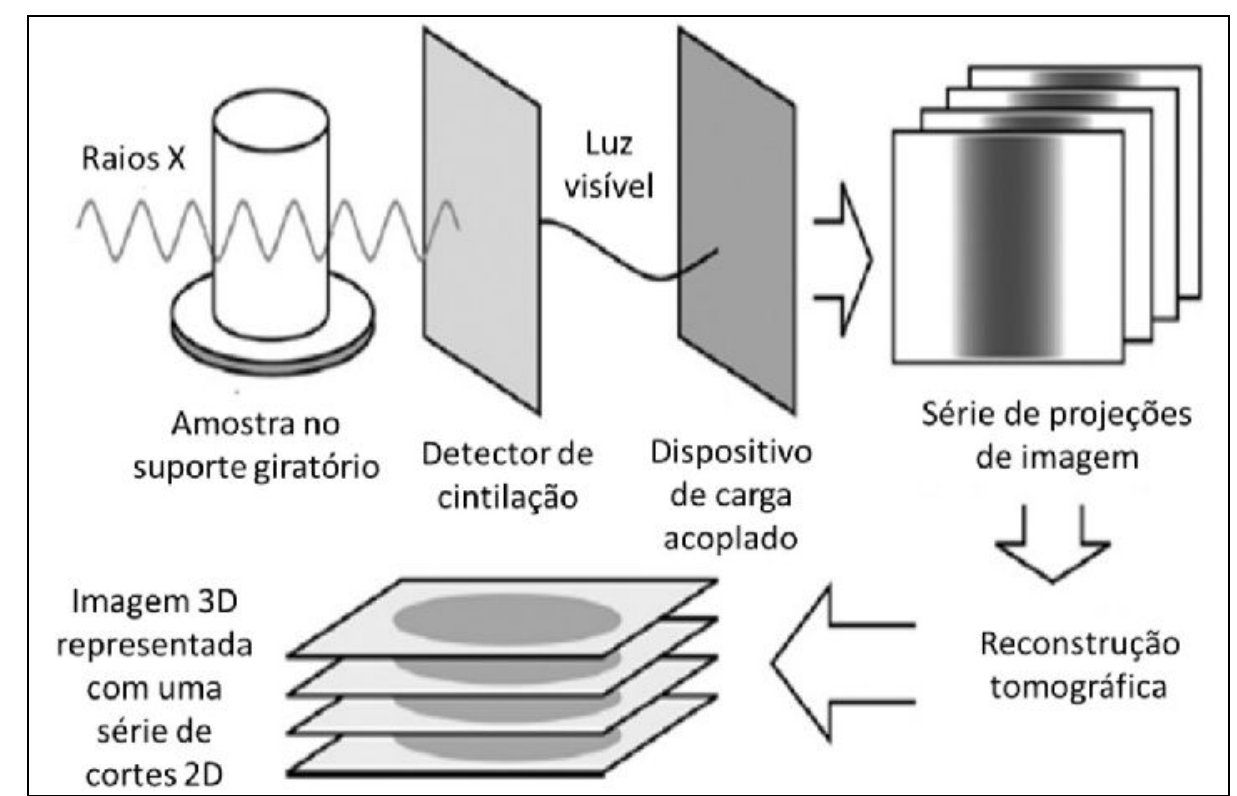

FIGURA 3 - Ilustração esquemática do processo de aquisição e reconstrução da imagem tridimensional em sistema TC ou microCT. Uma série de projeções de imagens é obtida e por meio de algoritmos matemáticos essas imagens são reconstruídas, produzindo um mapa tridimensional de diferentes pontos de absorção de raios X. Fonte: adaptado de Landis e Keane (2010).

\section{Sistemas de escaneamento microtomográfico da amostra}

Existem dois tipos de sistema de escaneamento microtomográfico. No primeiro, denominado de estrutura rotativa (rotating gantry), o microtomógrafo apresenta um emissor de radiação e um detector de raios $X$ alocados em uma estrutura que gira, sincronizadamente, em torno do eixo central da amostra examinada, a qual permanece imóvel (Figura 4 - A) (SCHAMBACH et al., 2010; CLARK; BADEA, 2014). Esse modelo de equipamento é conhecido como "Mini-TC", pois possuiu configuração análoga aos aparelhos de TC clínica e, portanto, é utilizado em avaliações in vivo de pequenos roedores (BADEA et al., 2008).

No segundo tipo de sistema de escaneamento, conhecido como amostra rotativa (rotating specimen), a amostra é rotacionada, enquanto a fonte e o detector de radiação permanecem fixos (Figura 4 - B) (CLARK; BADEA, 2014). Esse 
escaneamento é utilizado em amostras ex vivo, as quais foram obtidas de organismos vivos por meio de biópsias ou após a eutanásia do animal. Esse tipo de microtomógrafo não é adequado para experimentos com animais vivos, em virtude da necessidade de fixação do animal na posição vertical (BADEA et al., 2008; CLARK; BADEA, 2014) e mantê-lo imóvel durante a rotação, a fim de evitar que as imagens obtidas fiquem desfocadas (SCHAMBACH et al., 2010).

A

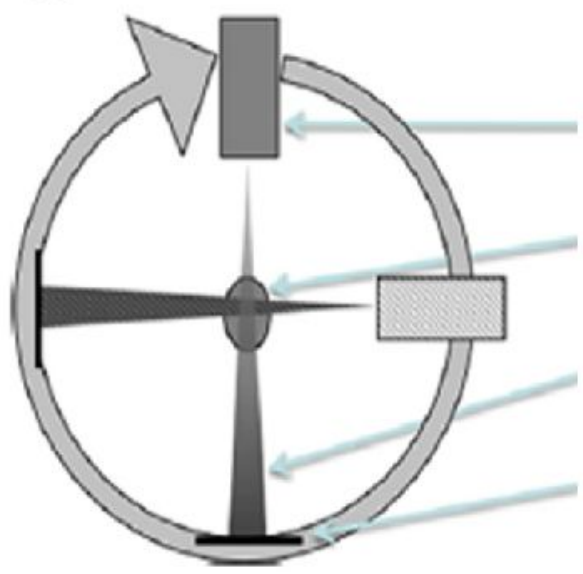

B

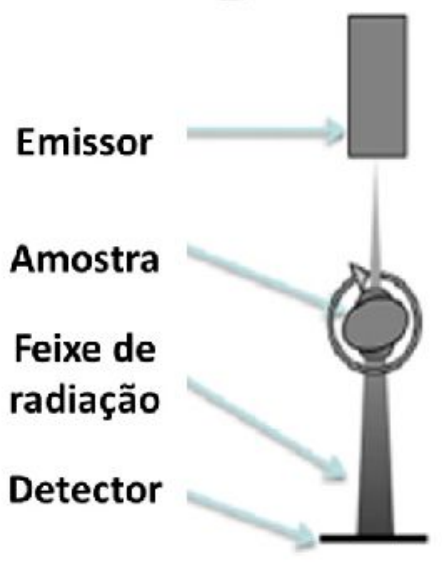

FIGURA 4 - Sistemas de escaneamento microtomográfico. A Estrutura rotativa. A amostra é posicionada no centro do equipamento e permanece imóvel, enquanto o emissor de radiação e o receptor de raios $X$ giram em torno da amostra. $B$ - Amostra rotativa. A amostra é fixada em um suporte que gira em $180^{\circ}$ a $360^{\circ}$, enquanto o emissor e o receptor de raio $X$ permanecem fixos. Fonte: adaptado de Schambach et al. (2010).

\section{APLICAÇÃO DA MICROCT EM AMOSTRAS BIOLÓGICAS}

A microCT tem sido utilizada na avaliação de vários tipos de tecidos biológicos, com destaque para estudos da microestrutura de ossos e dentes, pois são tecidos radiodensos e, portanto, facilmente visualizados. O exame microtomográfico de tecidos moles é mais difícil, pois são formados por elementos de baixo número atômico, como o carbono e o oxigênio. Dessa forma, esses tecidos apresentam pouco contraste em imagens microtomográficas, o que dificulta a visualização da estrutura interna dessas amostras (MIZUTANI; SUZUKI, 2012).

Esse empecilho pode ser resolvido pelo uso de contrastes radiodensos, como metais pesados, que apresentam alto número atômico e, quando impregnados na amostra, aumentam a atenuação do tecido marcado, possibilitando a visualização da microestrutura (BOERCKEL et al., 2014; BAIRD; TAYLOR, 2017). Os principais elementos utilizados como contrate em avaliações microtomográficas de amostras biológicas são ósmio, ouro, prata, iodo, platina, mercúrio, tungstênio e chumbo (MIZUTANI; SUZUKI, 2012). Nas próximas seções desta revisão serão apresentados resultados de estudos que utilizaram a microCT para a avaliação de amostras biológicas.

Osso

A utilização da microCT para avaliação da microestrutura óssea em animais e humanos tem crescido nos últimos anos. Atualmente essa técnica é considerada "padrão ouro" para a avaliação da morfologia e microarquitetura de ossos de ratos e 
de outros pequenos animais (BOUXSEIN et al., 2010). A microCT foi utilizada na avaliação das alterações ósseas provocadas pela osteoporose (BORAH et al., 2001) e osteoartrite (PATEL et al., 2003), na cicatrização óssea (TREJO-IRIARTE et al., 2019) e em alterações ósseas ocasionadas por fármacos (O’NEAL et al., 2010). Embora a avaliação histológica possibilite importantes informações sobre a celularidade e a dinâmica da remodelação óssea, essa técnica não permite o estudo da microarquitetura do tecido ósseo, visto que se limita a análise morfológica de alguns cortes. Diferentemente, a microCT possibilita a reconstrução tridimensional da microestrutura óssea (BOUXSEIN et al., 2010).

O grande número de publicações que utilizaram a microCT para a avaliação óssea, principalmente a partir dos anos 2000, associado a disponibilidade comercial de vários tipos de microtomógrafos, ocasionaram uma grande variabilidade nos métodos de obtenção de imagens, avaliação das amostras e descrição dos resultados. Consequentemente, ocorriam dificuldades na interpretação e comparação dos resultados dos estudos. Diante disso, foi disponibilizada uma padronização dos métodos de avaliação óssea por meio da microCT, que foi elaborada por especialistas na área. Esse trabalho contemplou recomendações sobre a padronização das terminologias e unidades, bem como as informações que devem ser incluídas na descrição metodológica (BOUXSEIN et al., 2010).

A microCT foi utilizada na avaliação dos efeitos do hiperparatiroidismo secundário renal (HSR) na microarquitetura óssea de ratos. Nesse estudo, foram obtidas imagens da metáfise proximal do fêmur desses animais. O HSR foi induzido por meio de nefrectomia parcial e fornecimento de dieta rica em fósforo. Após a eutanásia dos animais, amostras do fêmur foram submetidas ao exame microtomográfico e observou-se que os ratos com HSR apresentaram redução do volume ósseo trabecular e cortical, aumento da porosidade óssea e aumento da espessura das trabéculas (HOPPER et al., 2007) (Figura 5 - A, B, C e D).

Pesquisadores brasileiros avaliaram por meio da microCT, o efeito da suplementação com bório na qualidade óssea de ratos diabéticos. Nessa pesquisa, as imagens microtomográficas demonstraram que os ratos suplementados com bório apresentaram maior volume cortical e trabecular do fêmur em relação aos animais não suplementados (DESSORDI et al., 2017).

A avaliação por microCT tem sido amplamente utilizada no estudo de protocolos de reparação de fraturas ósseas, pois possibilita a identificação de alterações ósseas microestruturais e a avaliação da formação do calo ósseo (NYMAN et al., 2009). A microCT foi utilizada na avaliação do processo de cicatrização de fraturas do fêmur de ratos. Os autores dessa pesquisa concluíram que essa técnica é uma ferramenta sensível para a identificação de alterações na cicatrização óssea de fraturas, pois possibilita a mensuração do tamanho e densidade do calo ósseo. Dessa forma, a microCT pode ser utilizada como método auxiliar para a avaliação da cicatrização de fraturas de pequenos roedores como modelos experimentais (O’NEILL et al., 2012). 


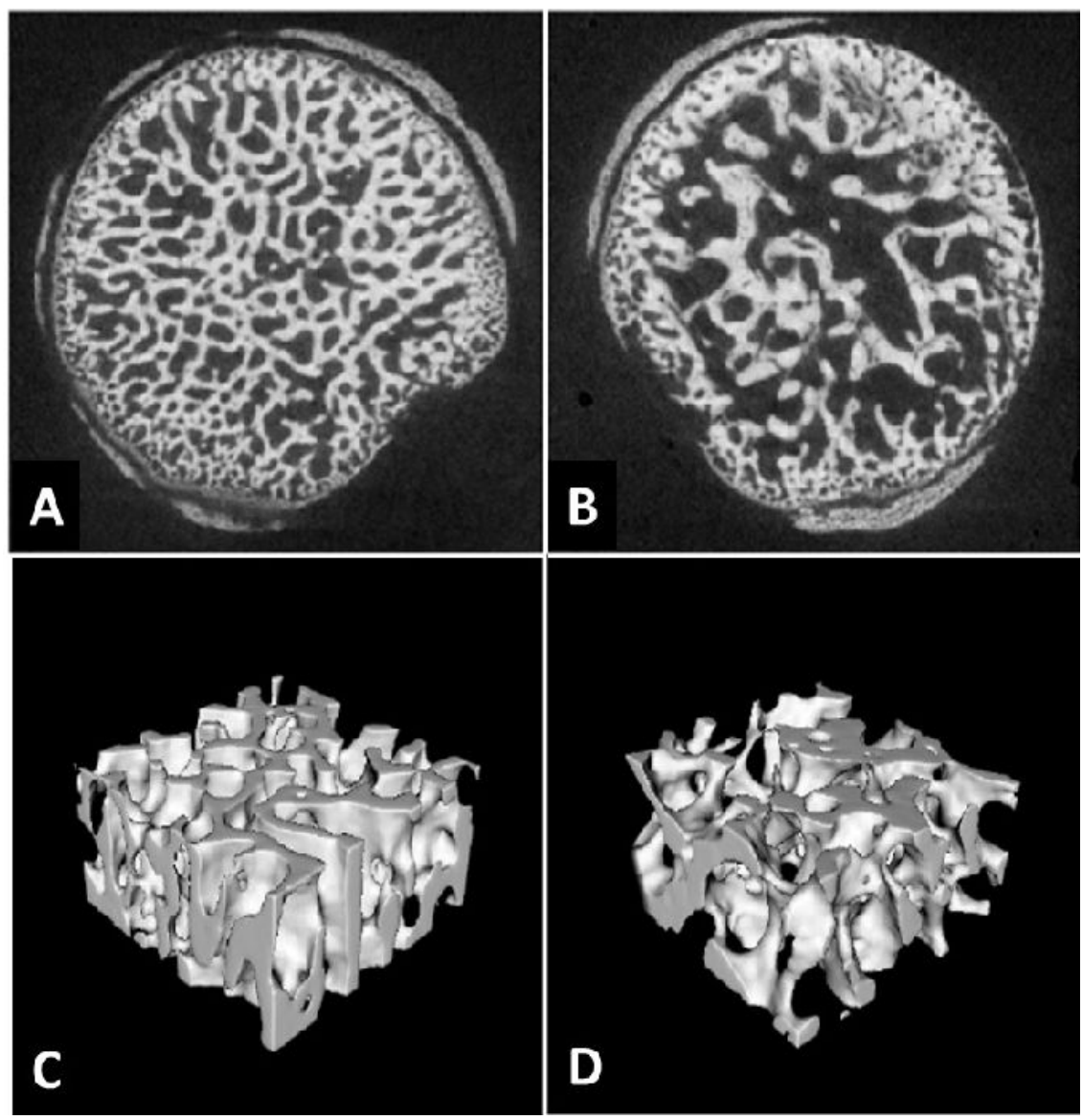

FIGURA 5 - Imagens microtomográficas bidimensionais (A e B) e trimensionais $(C$ e $D)$ da metáfise proximal do fêmur de ratos. A Imagem bidimensional da metáfise proximal do fêmur de animais do grupo controle mostrando a arquitetura do osso trabecular saudável. B - Imagem bidimensional da mesma região anatômica anterior, porém em ratos submetidos à nefrectomia parcial. Evidencia-se alterações significativas: as trabéculas estão consideravelmente mais espessas, os poros intratrabeculares apresentam-se aumentados e as trabéculas são menos numerosas em comparação ao grupo controle. C - Imagem tridimensional da metáfise proximal do fêmur de ratos do grupo controle que demonstra maior organização das trabéculas ósseas. D - Imagem tridimensional da mesma estrutura anatômica, porém em animais submetidos à nefrectomia parcial. Observa-se maior porosidade trabecular em comparação ao grupo controle (resolução 8,2 $\mu \mathrm{m}$ ). Fonte: Hopper et al. (2007).

Pesquisas envolvendo remodelações ósseas em modelos experimentais vivos também podem ser realizadas por meio da microCT. Pesquisadores canadenses validaram uma metodologia para examinar a concentração mineral óssea em lesões no crânio de ratos. Identificou-se que a microCT é uma técnica não invasiva que pode ser utilizada em experimentos de remodelação óssea in vivo, possibilitando uma avaliação rápida e precisa da concentração mineral óssea de lesões no crânio. Além disso, os autores sugeriram que esta técnica pode ser utilizada rotineiramente em pesquisas futuras sobre remodelação e crescimento ósseo em lesões no crânio de pequenos roedores. Dentre as vantagens da microCT nesse tipo de avaliação, destacam-se a obtenção de medidas quantitativas precisas, baixo coeficiente de variação e possibilidade de avaliações seriadas no mesmo animal (UMOH et al., 2009). 


\section{Dente}

A microCT é uma técnica que tem sido amplamente utilizada em pesquisas odontológicas. A utilização do exame microtomográfico em estudos odontológicos incluem a avaliação da concentração mineral do esmalte dentário e da dentina, identificação de desmineralização em áreas careadas, mensuração de cavidades dentárias, quantificação da dentina removida em diferentes tratamentos de cáries, avaliação da morfologia do canal radicular e identificação de microinfiltração em selantes dentários (NEVES et al., 2014; CHAŁAS et al., 2017).

A acurácia da microCT em amostras dentárias foi demonstrada em um estudo que comparou os resultados obtidos pelo exame microtomográfico, com outras técnicas de mensuração já consagradas, dentre elas: medição direta do dente com um paquímetro, medição do dente em imagens fotográficas e medição do dente por meio de imagem tridimensional obtida por um scanner de superfície. A microCT foi considerada um método confiável para realização de medições lineares em dentes (KIM et al., 2007).

A avaliação do esmalte dentário é considerada uma importante técnica para o estudo da evolução humana e é empregada em pesquisas em fósseis, fornecendo informações sobre taxonomia e filogenia de hominídeos (SWAIN; XUE, 2009). A acurácia da microCT na avaliação do esmalte dentário foi demonstrada em um estudo que comparou a microCT com a técnica de microscopia eletrônica de varredura. Amostras de dentes de primatas não humanos e humanos foram avaliadas utilizando-se as duas técnicas (Figura 6). Os resultados indicaram que o exame microtomográfico mensurou de forma precisa os parâmetros para o exame do esmalte dentário. Os autores encontraram uma variação de 3 a $5 \%$ entre as duas técnicas avaliadas (OLEJNICZAK; GRINE, 2006).

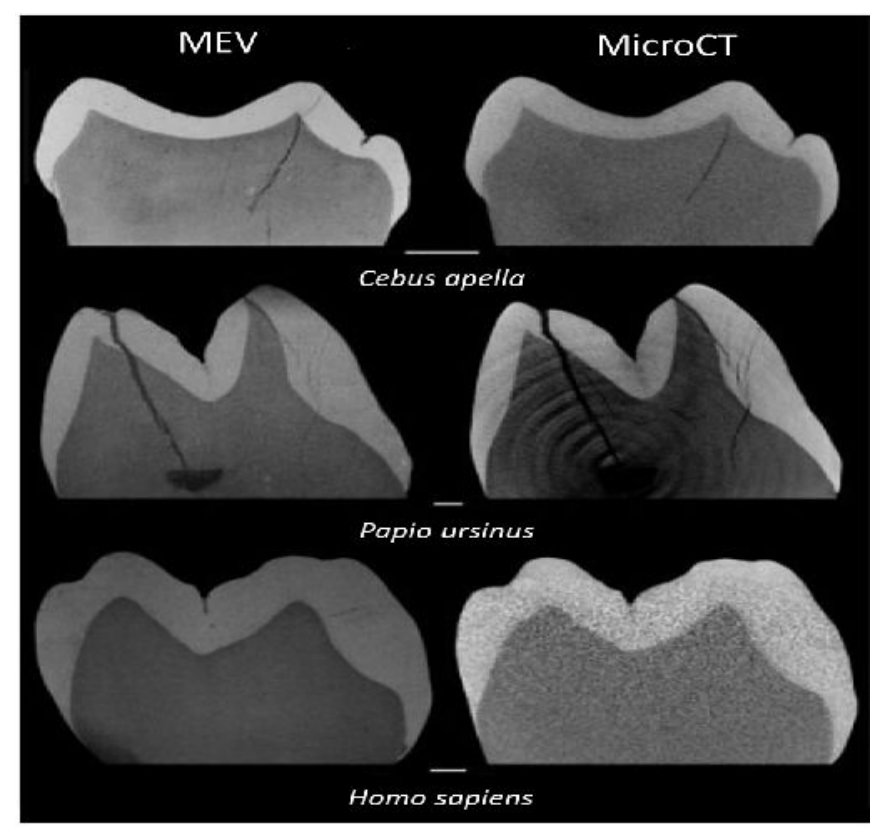

FIGURA 6 - Comparação entre as imagens obtidas por microscopia eletrônica de varredura (MEV) e microCT. As imagens apresentam o mesmo corte transversal de dentes molares de três primatas avaliados pelas duas técnicas. Cebus apela (macacoprego), Papio ursinus (babuíno) e Homo sapiens (humano). Barra de escala $1 \mathrm{~mm}$.

Fonte: adaptado de Olejniczak e Grine (2006). 


\section{Cartilagem}

A obtenção de imagens tridimensionais de tecido mole, como cartilagens, é um desafio, visto que a atenuação de raios $X$ nesse tipo de tecido é muito baixa. Dessa forma, é necessário o uso de contrastes para visualização dos tecidos. Entretanto, esses podem promover alterações nas características estruturais da amostra (NAVEH et al., 2014). Diante das alterações indesejadas na amostra ocasionadas pelo uso dos contrastes, foi descrita uma metodologia para a avaliação microtomográfica de tecidos cartilaginosos que não necessita do uso dessas substâncias. Utilizando-se uma câmara de posicionamento da amostra com ambiente altamente úmido (95\% umidade relativa), obteve-se imagens tridimensionais em alta resolução de tecido cartilaginosos, sem a utilização de contrastes. Os tecidos avaliados foram placa epifisária, disco intervertebral e ligamento periodontal. Segundo os autores desse estudo, o ar úmido absorve menos fótons de raios $\mathrm{X}$ e, consequentemente, promove melhora no contraste dos tecidos não corados (NAVEH et al., 2014).

Em contrapartida, para a avaliação de constituintes específicos da cartilagem por meio da microCT, faz se necessário o uso de contrastes. Em uma pesquisa, avaliou-se a eficiência do ácido fosfotúngstico (PTA) e do ácido fosfomolíbdico (PMA), ambos marcadores específicos de colágeno e atenuadores de raios $\mathrm{X}$, na visualização da distribuição de colágeno em amostras de cartilagem articular de equinos e humanos. Observou-se que a coloração com PTA possibilitou a reconstrução tridimensional de amostras osteocondrais incluindo a superfície da cartilagem articular. Assim, permitiu a avaliação da morfologia articular superficial e a identificação de alterações, como irregularidades e fissuras (NIEMINEN et al., 2015) (Figura 7).

\section{Avaliação cardiovascular}

A utilização de contrastes radiodensos em estudos angiográficos com a microCT possibilita a visualização de estruturas cardiovasculares em experimentos ex vivo e in vivo (BOERCKEL et al., 2014). Em um modelo experimental ex vivo, avaliou-se por meio da microCT as fases de recuperação vascular após uma injúria isquêmica no membro pélvico de camundongos. Após a eutanásia dos animais, a cavidade torácica foi aberta e injetou-se formol tamponado a $10 \%$ na veia cava caudal para fixação dos vasos. Após a retirada do formol, o contraste (Microfil $\Theta_{\text {, }}$ Flow Tech Inc., Carver-MA, EUA) foi aplicado nos vasos. Por meio da avaliação microtomográfica, observou-se a formação da circulação colateral frente a uma lesão isquêmica que, restaurou a capacidade de deambulação dos camundongos após quatro semanas da injúria (LANDÁZURI et al., 2012). As imagens microtomográficas da reparação vascular após injúria isquêmica no membro pélvico de camundongos podem ser observadas na Figura 8. 


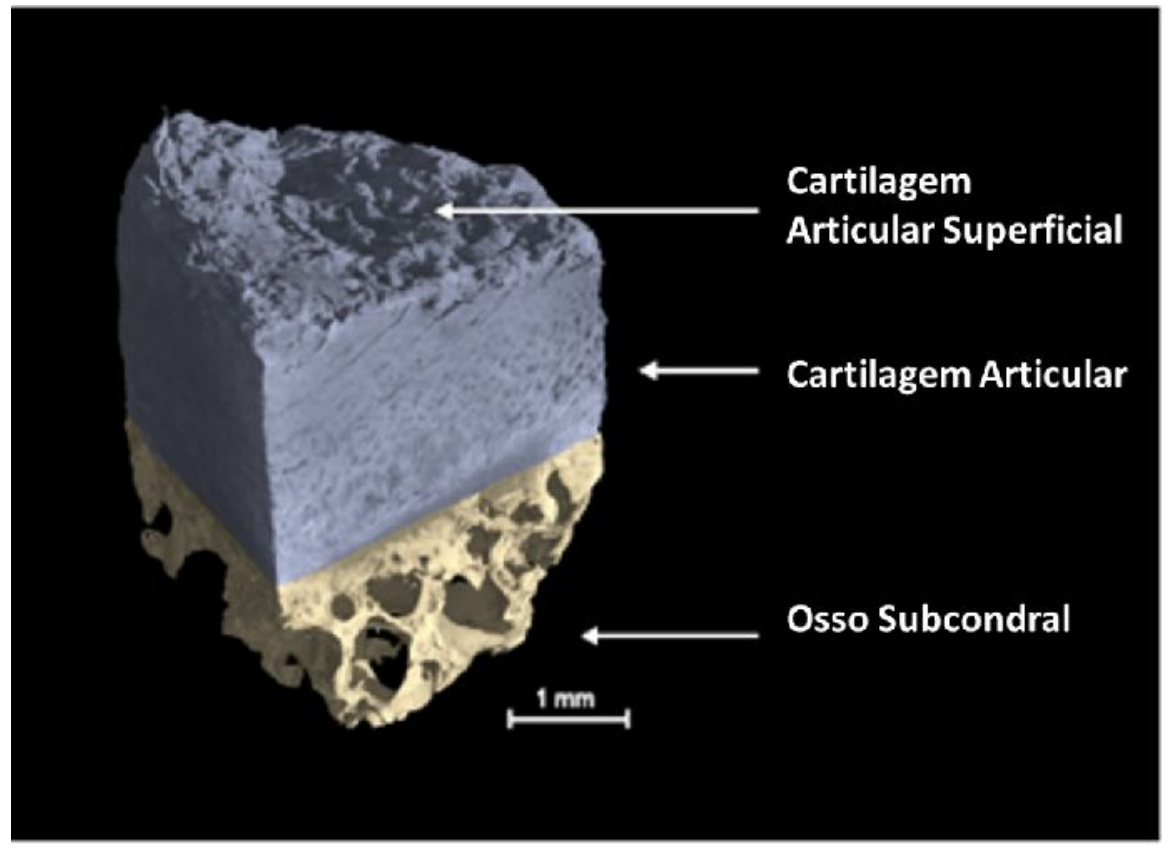

FIGURA 7 - Reconstrução tridimensional de uma amostra de cartilagem articular humana obtida por exame microtromográfico. A coloração com ácido fosfotúngstico (PTA) possibilitou a reconstrução tridimensional da amostra osteocondral incluindo a cartilagem articular superficial, onde observa-se a presença de irregularidades superficiais.

Fonte: adaptado de Nieminen et al. (2015).

A microCT também pode ser empregada na obtenção de imagens tridimensionais e em alta resolução em experimentos cardiovasculares in vivo com pequenos roedores. Essa técnica possibilita a identificação de alterações na anatomia vascular frente ao uso de protocolos terapêuticos em casos de doenças vasculares. A realização de uma angiografia em um microtomógrafo envolve várias etapas: a preparação do cateter intravenoso, anestesia do rato, colocação do cateter intravenoso, contenção física do animal no equipamento, preparação da bomba de infusão e escolha da configuração do sistema de microCT. Toda a descrição da técnica para a obtenção de imagens microtomográficas in vivo da vascularização abdominal e cerebral de pequenos roedores foi descrita por Schambach et al. (2010). 


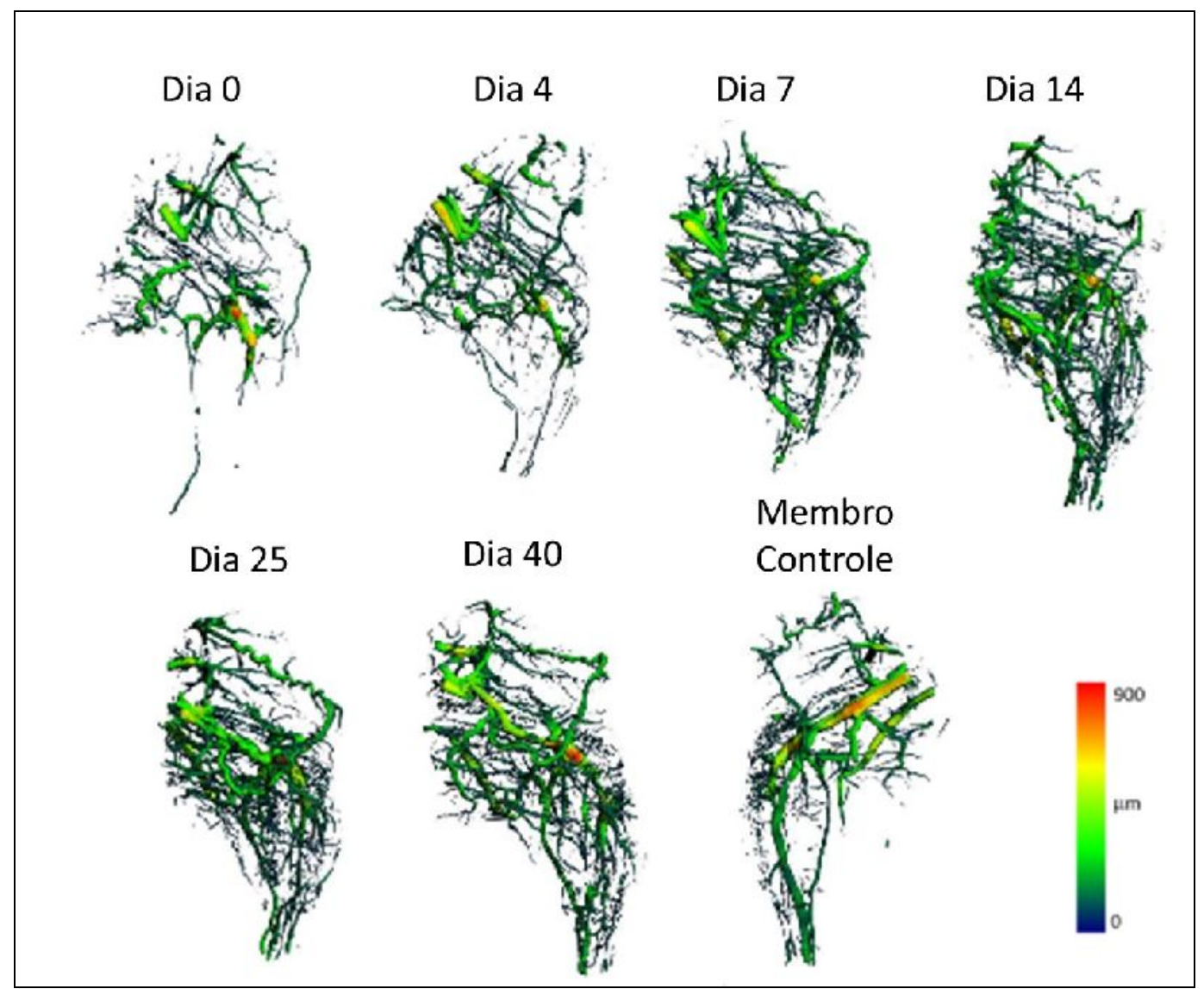

FIGURA 8 - Avaliação morfológica do desenvolvimento vascular após injúria isquêmica no membro pélvico de camundongos. Imagens representativas das avaliações por microCT do membro submetido a isquemia em vários momentos após a injúria isquêmica $\mathrm{A}$ escala representa o diâmetro dos vasos. Fonte: adaptado de Landázuri et al. (2012).

\section{Fígado}

As imagens tridimensionais obtidas pela microCT possibilitaram a visualização do fígado de camundongos com alta resolução espacial. A técnica permitiu a identificação da vascularização do fígado, bem como a identificação do volume e distribuição de lesões hepáticas. Entretanto, a identificação dessas lesões foi possível somente mediante o uso de contraste radiodenso para o tecido hepático ou específicos para neoplasias. Foram utilizados contrastes específicos para a microCT (Fenestra Liver Contraste ${ }^{\circledR}$, Advanced Research Technologies), que foram injetados na veia coccígea dos camundongos avaliados. O escaneamento foi realizado três horas após a administração do contraste e, por meio da reconstrução tridimensional, identificou-se as neoplasias no tecido hepático (Figura 9-A e B). Assim, foi possível avaliar a resposta a tratamentos com antineoplásicos por meio do acompanhamento da redução do volume dos tumores (MARTINIOVA et al., 2010). 


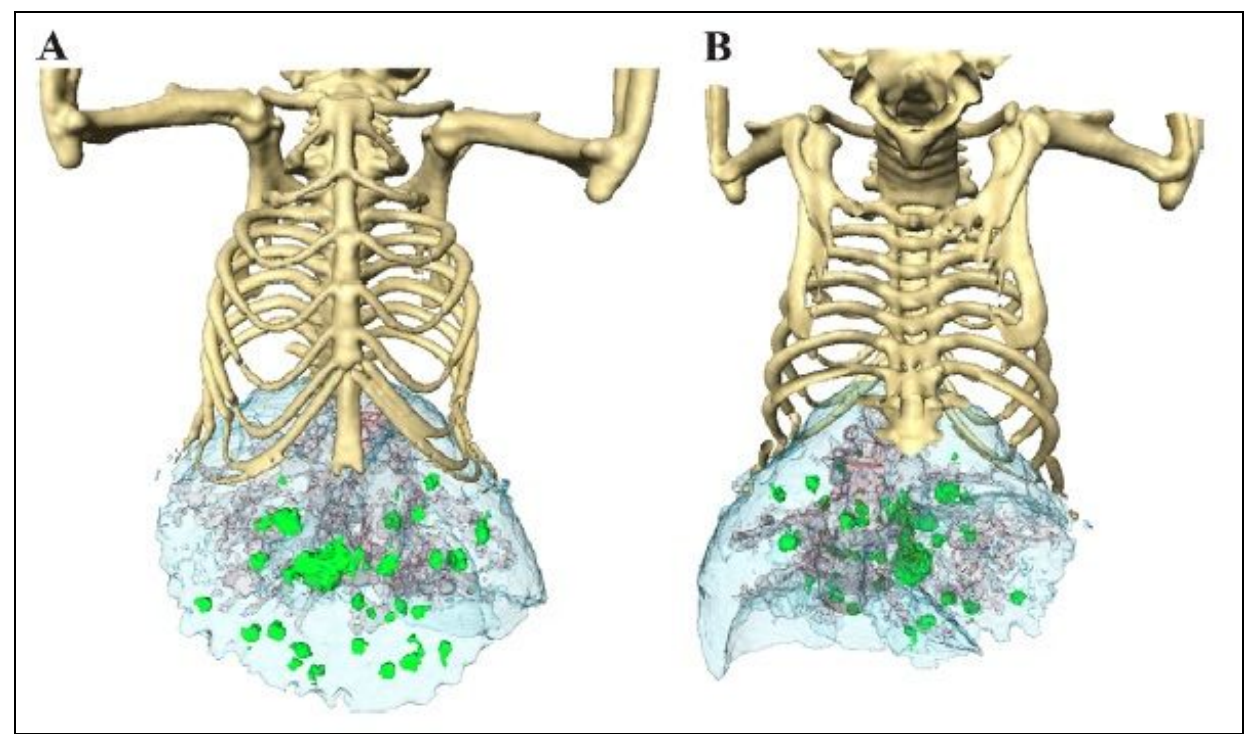

FIGURA 9 - Reconstrução tridimensional da avaliação por microCT do fígado de um rato com feocromocitoma metastático. Imagem anterior $(A)$ e posterior (B) do fígado de um rato com lesões neoplásicas 3 horas após a aplicação do contraste. Os lobos hepáticos (azul) estão bem definidos, assim como sua vascularização. Em virtude da diferença do tecido marcado pelo contraste e a densidade dele, é possível separar as neoplasias hepáticas (verde) da vascularização hepática (vermelho). Fonte: Martiniova et al. (2010).

\section{CONSIDERAÇÕES FINAIS}

A microCT é uma técnica de imagem não destrutiva, que possibilita a obtenção de imagens bidimensionais e tridimensionais da estrutura interna dos mais variados tipos de amostras. $O$ exame microtomográfico utiliza a atenuação de raios $X$ para a formação das imagens e possui os mesmos princípios físicos da TC, entretanto, é empregado na avaliação de pequenas amostras e no escaneamento de pequenos roedores. Dentre as vantagens da microCT estão a pequena necessidade de preparações da amostra e a possibilidade de realização de escaneamentos seriados sem alterações no material.

A microCT permite a reconstrução tridimensional da estrutura interna da amostra e gera dados quantitativos sobre essa estrutura. Dessa forma, possibilita análises amplas da morfometria tecidual em comparação à avaliação histológica e por microscopia eletrônica, as quais permitem o exame de apenas alguns cortes transversais. Acrescente-se aos pontos positivos da microCT que os equipamentos são compactos, não necessitam de grandes estruturas físicas para instalação e podem ser adquiridos comercialmente por centros de pesquisas e universidades. Quanto aos pontos negativos dessa técnica, pode-se citar o custo relativamente elevado para a aquisição do equipamento e a necessidade de um conhecimento sólido em física e computação, que é necessário para o máximo aproveitamento da técnica.

Atualmente, a microCT é amplamente utilizada em pesquisas biomédicas e é considerada a técnica de avaliação "padrão ouro" para o estudo de amostras de tecidos mineralizados, como osso e dente. Além disso, tem sido utilizada em estudos morfológicos de tecidos moles, como pulmão e fígado, obtidos por meio de biópsias ou após a eutanásia dos animais. Dentre as possibilidades de pesquisas com pequenos roedores em experimentos in vivo estão estudos com reparação tecidual, tratamento de neoplasias, engenharia de tecidos, alterações vasculares, dentre outros. 


\section{AGRADECIMENTOS}

Os autores agradecem ao Centro Nacional de Pesquisa em Energia e Materiais (CNPEM) e ao Laboratório Nacional de Nanotecnologia (LNNano), que possibilitaram o contato com a técnica de microtomografia computadorizada e a realização de análises. Os autores também agradecem à Fundação de Apoio a Pesquisa do Estado de Goiás (FAPEG) pela concessão de bolsa de estudo ao primeiro autor ( $\mathrm{n}-$ do processo 201710267000610).

\section{REFERÊNCIAS}

BADEA, C. T.; DRANGOVA, M.; HOLDWORTH, D. W.; JOHNSON, G. A. In vivo small animal imaging using micro-CT and digital subtraction angiography. Physics in medicine and biology, v. 53, n. 19, p. 319-350, 2008. Disponível em: <http://dx.doi.org/10.1088/0031-9155/53/19/R01>. doi: 10.1088/00319155/53/19/R01

BAIRD, E.; TAYLOR, G. X-ray micro computed-tomography. Current Biology, v. 27, n. 8, p. 289-291, 2017. Disponível em: <https://doi.org/10.1016/j.cub.2017.01.066>. doi: 10.1016/j.cub.2017.01.066.

BEAUCAGE, K. L.; POLLMANN, S. I.; SIMS, S. M.; DIXON, S. J.; HOLDSWORTH, D. W. Quantitative in vivo micro-computed tomography for assessment of agedependent changes in murine whole-body composition. Bone Reports, v. 5, p. 7080, 2016. Disponível em: <https://doi.org/10.1016/j.bonr.2016.04.002>. doi: 10.1016/j.bonr.2016.04.002.

BOERCKEL, J. D.; MASON, D. E.; MCDERMOTT, A. M.; ALSBERG, E. Microcomputed tomography: approaches and applications in bioengineering. Stem Cell Research \& Therapy, v. 5, n. 6, p. 144, 2014. Disponível em: <https://doi.org/10.1186/scrt534>. doi: 10.1186/scrt534.

BORAH, B.; GROSS, G. J.; DUFRESNE, T. E. SMITH, T. S. COCKMAN, M. D. et al. Three-dimensional microimaging (MRmicrol and microCT), finite element modeling, and rapid prototyping provide unique insights into bone architecture in osteoporosis. The Anatomical Record, v. 265, n. 2, p. 101-110, 2001. Disponíel em: <https://doi.org/10.1002/ar.1060>. doi: 10.1002/ar.1060.

BOUXSEIN, M. L.; BOYD, S. K.; CHRISTIANSEN, B. A.; GULDBERG, R. E.; JEPSEN K. K. J. et al. Guidelines for assessment of bone microstructure in rodents using micro-computed tomography. Journal of Bone and Mineral Research, v. 25, n. 7, p. 1468-1486, 2010. Disponível em: <https://doi.org/10.1002/jbmr.141>. doi: 10.1002/jbmr.141

CHAŁAS, R.; SZLAZZAK, K.; WÓJCIK-CHĘCIŃSKA, I.; JAROSZEWICZ, J.; MOLAK, $\mathrm{R}$. Observations of mineralised tissues of teeth in X-ray micro-computed tomography. Folia Morphologica (Poland), v. 76, n. 2, p. 143-148, 2017. Disponível em: < https://doi.org/10.5603/FM.a2016.0070 >. doi: 10.5603/FM.a2016.0070

CIERNIAK, R. X-ray computed tomography in biomedical engineering. vol. 2. London: Springer, 2011. $319 \mathrm{p}$. 
CLARK, D. P.; BADEA, C. T. Micro-CT of rodents: state-of-the-art and future perspectives. Physica medica, v. 30, n. 6, p. 619-634, 2014. Disponível em: <https://dx.doi.org/10.1016\%2Fj.ejmp.2014.05.011>.

doi: 10.1016\%2Fj.ejmp.2014.05.011

ÇIMEN, S.; GOOYA, A.; GRASS, M.; FRANGI, A. F. Reconstruction of coronary arteries from X-ray angiography: A review. Medical Image Analysis, v. 32, p. 46-68, 2016. Disponível em: <https://doi.org/10.1016/j.media.2016.02.007>. doi: 10.1016/j.media.2016.02.007

DALSTRA, M. CATTANEO, P. M.; LAURSEN, M. G.; BECKMANN, F.; MELSEN, B. Multi-level synchrotron radiation-based microtomography of the dental alveolus and its consequences for orthodontics. Journal of Biomechanics, v. 48, n. 5, p. 801806, 2015. Disponível em: <https://doi.org/10.1016/j.jbiomech.2014.12.014>. doi: 10.1016/j.jbiomech.2014.12.014

DESSORDI, R.; SPIRLANDELI, A. L.; ZAMARIOLI, A.; VOLPON, J. B.; NAVARRO, $M$. Boron supplementation improves bone health of non-obese diabetic mice. Journal of Trace Elements in Medicine and Biology, v. 39, p. 169-175, 2017. Disponível em: <https://doi.org/10.1016/j.jtemb.2016.09.011>. doi: 10.1016/j.jtemb.2016.09.011

DU PLESSIS, A.; BOSHOFF, W. P. A review of X-ray computed tomography of concrete and asphalt construction materials. Construction and Building Materials, v. 199, p. 637-651, 2019. Disponível em: <https://doi.org/10.1016/j.conbuildmat.2018.12.049>. doi: 10.1016/j.conbuildmat.2018.12.049

ELLIOTT, J. C.; DOVER, S. D. Three-dimensional distribution of mineral in bone at a resolution of 15 micron determined by $x$-ray microtomography. Metabolic Bone Disease \& Related Research, v. 5, n. 5, p. 219-221, 1984. Disponível em: <https://doi.org/10.1016/0221-8747(84)90062-6>. doi: 10.1016/0221-8747(84)900626.

ELLIOTT, J. C.; DOVER, S. D. X-ray microscopy using computerized axial tomography. Journal of Microscopy, v. 138, n. Pt 3, p. 329-331, 1985. Disponível em: <https://doi.org/10.1111/j.1365-2818.1985.tb02627.x>. doi: 10.1111/j.13652818.1985.tb02627.x

FERNANDES, J. S.; APPOLONI, C. R.; FERNANDES, C. P. Accuracy evaluation of an X-ray microtomography system. Micron, v. 85, p. 34-38, 2016. Disponível em: $<$ https://doi.org/10.1016/j.micron.2016.03.007>. doi: 10.1016/j.micron.2016.03.007

FREER, P. E.; WINKLER, N. Synthesized digital mammography Imaging. Radiologic Clinics of North America, v. 55, n. 3, p. 503-512, 2017. Disponível em: <https://doi.org/10.1016/j.rcl.2016.12.005>. doi: 10.1016/j.rcl.2016.12.005

HANKE, R.; FUCHS, T.; SALAMON, M.; ZABLER, S. X-ray microtomography for materials characterization. In: HÜBSCHEN, G.; ALTPETER, I.; TSCHUNCKY, R.; 
HERRMANN H-G (Eds.). Materials Characterization Using Nondestructive Evaluation (NDE) Methods. Cambridge: Woodhead Publishing, 2016. p. 45-79.

HESSENBRUCH, A. A brief history of x-rays. Endeavour, v. 26, n. 4, p.137-141, 2002. Disponível em: <https://doi.org/10.1016/S0160-9327(02)01465-5>. doi: $10.1016 / \mathrm{S} 0160-9327(02) 01465-5$

HOLDSWORTH, D. W.; THORNTON, M. M. Micro-CT in small animal and specimen imaging. Trends in Biotechnology, v. 20, n. 8, p. 34-39, 2002. Disponível em: < https://doi.org/10.1016/S0167-7799(02)02004-8>. doi: 10.1016/S01677799(02)02004-8

HOPPER, T. A.; WEHRLI, F. W.; SAHA, P. K.; ANDRE, J. B.; WRIGHT, A. C. et al. Quantitative microcomputed tomography assessment of intratrabecular, intertrabecular, and cortical bone architecture in a rat model of severe renal osteodystrophy. Journal of Computer Assisted Tomography, v. 31, n. 2, p. 320328, 2007. Disponível em: <https://doi.org/10.1097/01.rct.0000238007.19258.3d>. doi: 10.1097/01.rct.0000238007.19258.3d

ISAIAH, A.; PEREIRA, K. D. Laryngotracheal anomalies and airway fluoroscopy in infants. International Journal of Pediatric Otorhinolaryngology, v. 97, p. 109-112, 2017. Disponível em: <https://doi.org/10.1016/j.ijporl.2017.03.033>. doi: 10.1016/j.jporl.2017.03.033.

JACQUES, P. D.; NUMMER, A. R.; HECK, R. J.; MACHADO, R. The use of microtomography in structural geology: A new methodology to analyse fault faces. Journal of Structural Geology, v. 66, p. 347-355, 2014. Disponível em: <https://doi.org/10.1016/j.jsg.2014.06.004> doi: 10.1016/j.jsg.2014.06.004

KIM, I.; PAIK, K.-S.; LEE, S.-P. Quantitative evaluation of the accuracy of microcomputed tomography in tooth measurement. Clinical Anatomy, v. 20, n. 1, p. $27-$ 34, 2007. Disponível em: <http://dx.doi.org/10.1002/ca.20265>. doi: 10.1002/ca.20265

LANDÁZURI, N.; JOSEPH, G.; GULDBERG, R. E.; TAYLOR, W. R. Growth and regression of vasculature in healthy and diabetic mice after hindlimb ischemia. American Journal of Physiology. Regulatory, Integrative and Comparative Physiology, v. 303, n. 1, p. 48-56, 2012. Disponível em: <https://doi.org/10.1152/ajpregu.00002.2012>. doi: 10.1152/ajpregu.00002.2012

LANDIS, E. N.; KEANE, D. T. X-ray microtomography. Materials Characterization, v. $61, \quad$ n. 12, p. 1305-1316, 2010. Disponível em: < https://doi.org/10.1016/j.matchar.2010.09.012>. doi: 10.1016/j.matchar.2010.09.012

LEE, S.; CREAN, M. The story of radiology contents. Vienna, Austria: European Society of Radiology, 2013. 101p.

LI, H.; ZHANG, H.; TANG, Z.; HU, G. . Micro-computed tomography for small animal imaging: Technological details. Progress in Natural Science, v. 18, n. 5, p. 513- 
521, 2008. Disponível em: <https://doi.org/10.1016/j.pnsc.2008.01.002.> doi: 10.1016/j.pnsc.2008.01.002.

LOPES, A. P.; FIORI A. P.; REIS NETO, J. M.; MARCHESE, C.; VASCONCELLOS, E. M. G. et al. Análise tridimensional de rochas por meio de microtomografia computadorizada de raios $X$ integrada à petrografia. Geociências, v. 31, n. 1, p. 129-142, 2012. Disponível em: < http://www.ppegeo.igc.usp.br/index.php/GEOSP/article/view/7248>. Acesso em: 28 mar. 2019.

MACHADO, A. C.; LIMA, I.; LOPES, R. T. Effect of 3D computed microtomography resolution on reservoir rocks. Radiation Physics and Chemistry, v. 95, p. 405-407, 2014. Disponível em: <https://doi.org/10.1016/j.radphyschem.2012.12.029>. doi: 10.1016/j.radphyschem.2012.12.029.

MARTINIOVA, L.; SCHIMEL, D.; LAI, E. W.; LIMPUANGTHIP, A. KVETNANSKY R. et al. In vivo micro-CT imaging of liver lesions in small animal models. Methods, $v$. 50, n. $1, \quad$ p. 20-25, 2010. Disponível em: <https://doi.org/10.1016/j.ymeth.2009.05.016>. doi: 10.1016/j.ymeth.2009.05.016.

METSÄLÄ, E.; FRIDELL, K. Insights into the methodology of radiography science. Radiography, v. 24, n. 4, p. 105- 108, 2018. Disponível em: <https://doi.org/10.1016/j.radi.2018.05.010>. doi: 10.1016/j.radi.2018.05.010

MIZUTANI, R.; SUZUKI, Y. X-ray microtomography in biology. Micron, v. 43, n. 2, p. 104-115, 2012. Disponível em: <https://doi.org/10.1016/j.micron.2011.10.002.>. doi: 10.1016/j.micron.2011.10.002.

MOHAN, G.; PERILLI E.; KULIWABA, J. S.; HUMPHRIES, J. M., PARKINSON, I. H. et al. Application of in vivo micro-computed tomography in the temporal characterisation of subchondral bone architecture in a rat model of low-dose monosodium iodoacetate-induced osteoarthritis. Arthritis Research \& Therapy, v. 13, n. 6, p. R210, 2011. Disponível em: <https://dx.doi.org/10.1186\%2Far3543>. doi: $10.1186 \% 2 F a r 3543$

NAVEH, G. R.; BRUMFELD, V.; DEAN, M.; SHAHAR, R.; WEINER, S. Direct microCT imaging of non-mineralized connective tissues at high resolution. Connective Tissue Research, v. 55, n. 1, p. 52-60, 2014. Disponível em: $<$ https://doi.org/10.3109/03008207.2013.867333>.

doi: $10.3109 / 03008207.2013 .867333$

NEVES, A. A.; JAECQUES, S.; VAN ENDE, A.; CARDOSO, M. V.; COUTINHO, E. et al. 3D-microleakage assessment of adhesive interfaces: exploratory findings by $\mu \mathrm{CT}$. Dental Materials, v. 30, n. 8, p. 799-807, 2014. Disponível em: <https://doi.org/10.1016/j.dental.2014.05.003>. doi: 10.1016/j.dental.2014.05.003

NIEMINEN, H. J.; YLITALO, T.; KARHULA, S.; SUURONEN, J. P.; KAUPPINEN, S. et al. Determining collagen distribution in articular cartilage using contrast-enhanced micro-computed tomography. Osteoarthritis and Cartilage, v. 23, n. 9, p. 1613- 
1621, 2015. Disponível em: <http://dx.doi.org/10.1016/j.joca.2015.05.004> doi: 10.1016/j.joca.2015.05.004

NYMAN, J. S.; MUNOZ, S.; JADHAV, S.; MANSOUR, A.; YOSHII, T. et al. Quantitative measures of femoral fracture repair in rats derived by micro-computed tomography. Journal of Biomechanics, v. 42, n. 7, p. 891-897, 2009. Disponível em: <https://doi.org/10.1016/j.jbiomech.2009.01.016>. doi: 10.1016/j.jbiomech.2009.01.016

O'NEAL, J. M.; DIAB, T.; ALLEN, M. R.; VIDAKOVIC, B.; BURR, D. B. et al. One year of alendronate treatment lowers microstructural stresses associated with trabecular microdamage initiation. Bone, v. 47, n. 2, p. 241-247, 2010. Disponível em: <https://doi.org/10.1016/j.bone.2010.05.016>. doi: 10.1016/j.bone.2010.05.016

O'NEILL, K. R.; STUTZ, C. M.; MIGNEMI, N. A.; BURNS, M. C.; MURRY, M. R. et al. Micro-computed tomography assessment of the progression of fracture healing in mice. Bone, v. 50, n. 6, p. 1357-1367, 2012. Disponível em: <http://dx.doi.org/10.1016/j.bone.2012.03.008>. doi: 10.1016/j.bone.2012.03.008

OLEJNICZAK, A. J.; GRINE, F. E. Assessment of the accuracy of dental enamel thickness measurements using microfocal X-ray computed tomography. The Anatomical Record, v. 288, n. 3, p. 263-275, 2006. Disponível em: <https://doi.org/10.1002/ar.a.20307>. doi: 10.1002/ar.a.20307

PATEL, V.; ISSEVER, A. S.; BURGHARDT, A.; LAIB, A.; RIES, M. et al. MicroCT evaluation of normal and osteoarthritic bone structure in human knee specimens. Journal of Orthopaedic Research, v. 21, n. 1, p. 6-13, 2003. Disponível em: <https://doi.org/10.1016/S0736-0266(02)00093-1>. doi: 10.1016/S07360266(02)00093-1

ROBERTO-RODRIGUES, M.; FERNANDES, R. M.; SENOS, R.; SCORALICK, A. C.; BASTOS, A. A. et al. Novel rat model of nonunion fracture with vascular deficit. Injury, v. 46, n. 4, p. 649-654, 2015. Disponível em: <https://doi.org/10.1016/j.injury.2015.01.033>. doi: 10.1016/j.injury.2015.01.033

SCHAMBACH, S. J.; BAG, S.; GRODEN, C.; SCHILING, L.; BROCKMANN, M. A. Vascular imaging in small rodents using micro-CT. Methods, v. 50 , n. 1, p. 26-35, 2010. Disponível em: <https://doi.org/10.1016/j.ymeth.2009.09.003>. doi: 10.1016/j.ymeth.2009.09.003

SCHICHOR, C.; TERPOLILLI, N.; THORSTEINSDOTTIR, J.; TONN, J-C. Intraoperative computed tomography in cranial neurosurgery. Neurosurgery Clinics of North America, v. 28, n. 4, p. 595-602, 2017. Disponível em: <https://doi.org/10.1016/j.nec.2017.05.010>. doi: 10.1016/j.nec.2017.05.010.

STOCK, S. R. X-ray microtomography of materials. International Materials Reviews, v. 44, n. 4, p. 141-164, 1999. Disponível em: <http://dx.doi.org/10.1179/095066099101528261>. doi: $10.1179 / 095066099101528261$ 
SWAIN, M. V.; XUE, J. State of the art of Micro-CT applications in dental research. International Journal of Oral Science, v. 1, n. 4, p. 177-188, 2009. Disponível em: <https://doi.org/10.4248/IJOS09031>. doi: 10.4248/IJOS09031.

TREJO-IRIARTE, C. G.; SERRANO-BELLO, J.; GUTIÉRREZ-ESCALONA, R.; MERCADO-MARQUES, C.; GARCÍA-HONDUVILLA, N. et al. Evaluation of bone regeneration in a critical size cortical bone defect in rat mandible using microCT and histological analysis. Archives of Oral Biology, 2019. Disponível em: <https://doi.org/10.1016/j.archoralbio.2019.01.010>. 10.1016/j.archoralbio.2019.01.010

UMOH, J. U.; SAMPAIO, A. V.; PITELKA, V.; GOLDBERG, H. A.; UNDERHILL, T. M. et al. In vivo micro-CT analysis of bone remodeling in a rat calvarial defect model. Physics in Medicine and Biology, v. 54, n. 7, p. 2147-2161, 2009. Disponível em: <https://doi.org/10.1088/0031-9155/54/7/020>. doi: 10.1088/0031-9155/54/7/020

ZANOLLI, C.; SCHILLINGER B.; BEAUDET, A.; KULLMER, O.; MACCHIARELLI, R. et al. Exploring hominin and non-hominin primate dental fossil remains with neutron microtomography. Physics Procedia, v. 88, p. 109-115, 2017. Disponível em: $<$ https://doi.org/10.1016/j.phpro.2017.06.014>. doi: 10.1016/j.phpro.2017.06.014 